\title{
EFFECT PROCESSING VARIABLES ON THE CHARACTERISTICS OF ITRACONAZOLE HOLLOW MICROSPHERES
}

\author{
SURBHI ROHILLA*, D. C. BHATT, SHAVETA AHALWAT
}

Department of Pharmaceutical Sciences, Guru Jambheshwar University of Science and Technology, Hisar, Haryana 125001, India Email: surbhiraman.rohilla22@gmail.com

Received: 26 Jul 2019, Revised and Accepted: 23 Sep 2019

\begin{abstract}
Objective: The purpose of the study was to develop the multiple unit non-effervescent gastroretentive floating hollow microspheres to enhance the bioavailability of the drug by varying the concentration of low-density polymer and release modifier to retaining the formulation at its absorption
\end{abstract} site. Design of experiment approach applied to get the best possible formulation with minimum assets and experimentation.

Methods: The hollow microspheres were prepared by emulsion solvent diffusion-evaporation technique using ethylcellulose as low-density polymer and Eudragit E100 as release modifier. The central composite design was used for the optimization of independent variables and was evaluated for particle size, entrapment efficiency, in vitro floating ability and drug release characteristics.

Results: The physicochemical analysis was done to confirm any interaction between drug and excipients. The Scanning Electron Microscopy (SEM) showed a smooth, spherical surface with an inner hollow cavity. The stability study proves that the hollow microspheres were more stable under different storage conditions with no significant changes in formulation. The drug release mechanism of the optimized batch can be explained by Korsmeyer Peppas model.

Conclusion: Based on the results, the hollow microspheres with a release modifying polymer offers a superior approach to retain the formulation in the stomach.

Keywords: Floating hollow microspheres, Itraconazole, Stomach specific delivery

(C) 2019 The Authors. Published by Innovare Academic Sciences Pvt Ltd. This is an open access article under the CC BY license (http://creativecommons.org/licenses/by/4.0/) DOI: http://dx.doi.org/10.22159/ijap.2019v11i6.35098

\section{INTRODUCTION}

Itraconazole incompletely absorbed from the gastrointestinal tract. It is well absorbed from the upper part of GIT, possibly due to a weak basic property having ionization constant (PKa) 3.7 and is highly lipophilic $[1,2]$. This narrow absorption window is responsible for its low bioavailability, unpredictable absorption and inter and intrasubject variability [3]. The plasma drug concentration should be above minimum effective concentration for desired pharmacological action. In the case of immuno-compromised patients the plasma concentration lower then minimum effective concentration may results in a relapse of disease due to poor therapeutic effect [4]. Several attempts have been made to improve the oral bioavailability of itraconazole such as floating gastroretentive tablets [5], mucoadhesive sustained-release tablet by compression of solid dispersion [6], self-emulsifying formulation [7], solid dispersion [8], mixed polymeric micellar formulation [9].

Itraconazole has a narrow absorption window in the upper gastrointestinal tract which represents a rationale to develop a gastroretentive dosage form to provide continuous delivery of drug at its absorption site. To increase the absorption of the drug, it is very important to know the absorption site of drug. Various gastroretentive formulations have been studied in last few decades such as bioadhesive systems; floatation systems; high-density systems; magnetic systems; swellable systems; and super porous hydrogel systems to prolong the stomach retention of the dosage form. The floating dosage forms are the most reliable and economical among several approaches to increase the gastric retention and to alter the properties of the drug in a beneficial way [10-12]. It can be categorized in a single and multiple-unit floating system. Multiple unit floating system is better suited as it reduces the risk of local irritation of stomach wall and also avoids "all or none" effect which lowers the dose-dumping effect thereby reduces the inter-subject variability [13].

Ethylcellulose is a cellulose derivative also known as non-ionic ethyl ether of cellulose [14]. The low density of ethylcellulose makes it the most commonly used polymer for the preparation of floating microspheres. It is a biocompatible, biodegradable hydrophobic polymer $[15,16]$. So, to overcome the limitation there is a need to add polymer which facilitates the ingression of dissolution medium to craft channels in polymer matrix by increasing wet ability of hydrophobic polymer and provide rapid diffusion of drug and more drug release $[17,18]$. Eudragit E100 is a pH-dependent cationic polymer, having solubility in gastric fluid and can swell at pH 5.0 [19].

The present investigation has a rationale to formulate floating microspheres of itraconazole for the enhancement of bioavailability and gastric residence time and design of experiment approach was used to study the effect of various process variables. The effect of the ratio of low-density polymer and release modifier and concentration of emulsifier on particle size, entrapment efficiency, drug release, buoyancy and other physicochemical properties of floating microspheres.

\section{MATERIALS AND METHODS}

\section{Materials}

Itraconazole was received as a gift sample from Zydus Cadila Healthcare Limited, India. Eudragit E100 was received as a gift sample from Evonik Degussa India Pvt. Ltd, Mumbai, India. Ethyl Cellulose, Dichloromethane, Polyvinyl alcohol (PVA) and Tween 80 were purchased from Thomas baker Pvt. Ltd., New Delhi. All other reagents and solvents used were of analytical grade and used without further modification.

\section{Process conditions for floating microspheres}

Hollow microspheres were prepared by using the emulsion solvent diffusion evaporation method with slight modification from previously reported methods [20-22]. Ethylcellulose was used as low-density matrix-forming polymer and Eudragit E100 was used as release modifying polymer. Dichloromethane and ethanol were used as an organic solvent and PVA solution in distilled water was used as an aqueous surfactant phase. The investigated process variables that may affect the microsphere preparation, including temperature (room temperature, 40,60), stirring speed (250 and $300 \mathrm{rpm}$ ), and 
stirring time (30 min, $1 \mathrm{~h}$, and $2 \mathrm{~h}$ ). The Floating microsphere preparation describes in the following text.

\section{Preparation of microspheres}

The non-effervescent hollow microspheres containing itraconazole were prepared by using the above-mentioned method. Drug, ethylcellulose and Eudragit E100 were co-dissolved in dichloromethane and ethanol mixture (1:1). The resultant slurry was then introduced slowly in a drop-by-drop manner to $30 \mathrm{ml}$ PVA solution containing $0.2 \% \mathrm{w} / \mathrm{v}$ Tween- 80 while being stirred at 350 rpm using a three-blade propeller-type agitator for $2 \mathrm{~h}$ at $40^{\circ} \mathrm{C}$. The polymeric floating microspheres were formed by diffusion and subsequent evaporation of solvent mixture. The system temperature was maintained constant throughout the process to evaporate the solvent. The formed microspheres were harvested by filtration and washed three times with distilled water. The collected hollow microspheres were dried at room temperature for $24 \mathrm{~h}$ and stored in desiccator $[23,24]$.

\section{Design of experiment}

A central composite design was applied to design the experiment to optimize the responses and optimum process parameters. The Polymers ratio $\left(\mathrm{X}_{1}\right)$ and concentration of PVA $\left(\mathrm{X}_{2}\right)$ were selected as independent variables, whereas particle size $\left(\mathrm{R}_{1}\right)$, drug entrapment efficiency $\left(R_{2}\right)$, percentage buoyancy $\left(R_{3}\right)$ and percent cumulative drug release $\left(\mathrm{R}_{4}\right)$ were kept as dependent variables. Each factor was studies at 3 levels $(-1,0,+1)$; the experimental design layout suggested thirteen runs. Statistical analysis was performed and polynomial equations for each response variable was generated using Design-Expert software ${ }^{\circledR}$ (Version 11.0.0.5, Stat-ease Inc., Minneapolis, MN). The design matrix including investigated responses is presented in table 1 .

Table 1: Process parameters and response values of central composite design

\begin{tabular}{|c|c|c|c|c|c|c|}
\hline Code & $\begin{array}{l}\text { Polymer ratio } \\
\text { (Eudragit E100 : EC) }\end{array}$ & $\begin{array}{l}\text { Concentration of PVA } \\
(\% w / v)\end{array}$ & $\begin{array}{l}\text { Particle } \\
\text { Size }(\mu \mathrm{m})\end{array}$ & $\begin{array}{l}\text { Entrapment efficiency } \\
(\%)\end{array}$ & $\begin{array}{l}\text { Buoyancy } \\
(\%)\end{array}$ & $\begin{array}{l}\text { Cumulative drug release } \\
\text { (\%) }\end{array}$ \\
\hline F1 & $0(1: 4)$ & $0(0.75)$ & 336.1 & 72.2 & 52.52 & 87.11 \\
\hline $\mathrm{F} 2$ & $-1.414(1: 1.68)$ & $0(0.75)$ & 305 & 83.67 & 53.31 & 94.43 \\
\hline F3 & $0(1: 4)$ & $0(0.75)$ & 352.2 & 74.79 & 52.66 & 84.81 \\
\hline $\mathrm{F} 4$ & $0(1: 4)$ & $0(0.75)$ & 383.2 & 76.35 & 56.05 & 86.15 \\
\hline F5 & $1(1: 6)$ & $-1(0.5)$ & 502.4 & 89.83 & 78.59 & 77.78 \\
\hline F6 & $1(1: 6)$ & $1(1.0)$ & 424.3 & 81.36 & 56.97 & 82.33 \\
\hline F7 & $0(1: 4)$ & $0(0.75)$ & 374.2 & 75.12 & 54.25 & 87.59 \\
\hline F8 & $-1(1: 2)$ & $1(1.0)$ & 332.2 & 79.05 & 45.43 & 92.5 \\
\hline F9 & $0(1: 4)$ & $1.414(1.1)$ & 295.7 & 80.89 & 50.85 & 90.45 \\
\hline F10 & $-1(1: 2)$ & $-1(0.5)$ & 432.1 & 84.11 & 60.77 & 87.72 \\
\hline F11 & $1.414(1: 8.68)$ & $0(0.75)$ & 447 & 86.46 & 69.58 & 74.26 \\
\hline F12 & $0(1: 4)$ & $0(0.75)$ & 395.2 & 75.87 & 57.31 & 84.48 \\
\hline F13 & $0(1: 4)$ & $-1.414(0.4)$ & 482.6 & 93.74 & 72.58 & 79.15 \\
\hline
\end{tabular}

\section{Characterization of floating microspheres}

\section{Physico-chemical analysis}

Fourier Transform Infra-Red spectroscopy analysis (FTIR) and thermal analysis were done to determine any possible chemical interaction between drug and excipients. For FTIR analysis, the samples were finely powdered and mixed with $\mathrm{KBr}$ and scanned using an FTIR spectrophotometer (IR Affinity, Shimadzu) in the wavelength region between 4000 and $400 \mathrm{~cm}^{-1}$.

The thermal analysis of samples was done by Differential Scanning Calorimetry (DSC) by Differential Scanning Calorimeter (DSC 25, TA instruments). Samples of 2.5-5 mg were placed into an aluminum pan and heated at a constant rate $\left(10^{\circ} \mathrm{C} \mathrm{min}^{-1}\right)$, from 30 to $300{ }^{\circ} \mathrm{C}$ under nitrogen atmosphere.

Powder X-ray diffraction analysis was done to monitor the nature of the pure drug and after encapsulation into microspheres by X-ray diffractometer (Rigaku Miniflex-II X-ray diffractometer). The data set was collected in continuous scan at step size $0.04^{\circ} 2 \theta$ and angle range of $10^{\circ}-70^{\circ}$.

\section{Particle size and particle surface morphology}

Particle Size analysis was done using the mastersizer (Microtrac S3500 , USA) equipped with a liquid handling system while the morphology of particles was observed by SEM.

\section{Drug content}

For determining the proportion of the drug that got entrapped in microspheres, $10 \mathrm{mg}$ of microspheres containing drug was dissolved in methanol. The samples were filtered through a $0.45 \mu \mathrm{m}$ membrane and after appropriate dilution; the samples were assayed using UV-visible spectrophotometer (Cary 5000, Varian, Australia) at $262 \mathrm{~nm}$ against blank methanol containing the same quantity of blank microspheres. The drug content of floating microspheres can be calculated by dividing the weight of the drug in microspheres by weight of microspheres [25].

\section{Drug entrapment efficiency}

The drug entrapment efficiency in floating microspheres can be estimated by dissolving a predetermined amount of microspheres in methanol, filter the samples and analyze spectrophotometrically after appropriate dilution if required $[25,26]$.

$$
\% \text { Entrapment Efficiency }=\frac{\text { Practical drug content }}{\text { theoretical drug content }} \times 100
$$

\section{In vitro floating ability}

This study is carried out by using USP Type I dissolution apparatus stirred at $100 \mathrm{rpm}$ at $37 \pm 0.5{ }^{\circ} \mathrm{C}$. Weight amount of floating microspheres were spread on the surface of simulated gastric fluid (pH 1.2) containing surfactant. After a specified period, both the floating and settled fraction of microspheres were collected separately, dried and weighed [27]. The floating ability or percent buoyancy was calculated by the given formula

$$
\text { \%Buoyancy }=\frac{\text { Weight of hollow microspheres }}{\text { Total weight of hollow and settled microsphere }} \times 100
$$

\section{In vitro drug release study}

The drug release rate from microspheres was carried out using USP dissolution apparatus II. Microspheres equivalent to $30 \mathrm{mg}$ of the drug were treated with $900 \mathrm{ml}$ simulated gastric fluid $(\mathrm{pH}$ 1.2) containing $0.5 \% \mathrm{w} / \mathrm{v}$ tween 80 having a paddle rotation speed of $100 \mathrm{rpm}$ at $37 \pm 0.5^{\circ} \mathrm{C}$. The samples were withdrawn at regular time intervals and replaced it with fresh medium to maintain the sink conditions, then the samples were passed through a $0.45 \mu \mathrm{m}$ membrane filter, and analyzed spectrophotometrically at $258 \mathrm{~nm}$. All experiments were carried out in triplicate.

\section{Drug release kinetics}

The mechanism of drug release from microspheres were determined by applying different kinetic models to the data obtained from in vitro analysis such as Zero-order kinetics (percent release vs. time), first-order kinetics (log percent release vs. time), Higuchi's model 
(percent release vs. square root of time), Korsemeyer and Peppa's model (log cumulative percentage drug release vs. log time) $[28,29]$.

\section{Stability studies}

The optimized formulation was tested for stability profile at normal and accelerated conditions as per ICH guidelines [30]. The formulation was placed separately in borosilicate screw-capped glass container and stored at normal room temperature $\left(25 \pm 2{ }^{\circ} \mathrm{C}\right)$, freezing temperature $\left(5 \pm 3{ }^{\circ} \mathrm{C}\right)$ and under accelerated conditions $\left(40 \pm 2{ }^{\circ} \mathrm{C} / 75 \pm 5 \% \mathrm{RH}\right)$ for $6 \mathrm{mo}$. After predetermined time intervals, the samples were evaluated for visual physical appearance, Drug content, buoyancy and drug release studies [31, 32].

\section{RESULTS AND DISCUSSION}

\section{Effect of process conditions}

Solvent evaporation technique is affected by a large number of process variables. In the present study, the effect of process variables such as stirring rate, system temperature and stirring time was observed over percent yield and physical appearance of floating microspheres. For that $400 \mathrm{mg}$ of ethyl cellulose was used as lowdensity polymer and $100 \mathrm{mg}$ of Eudragit E100 polymer was used as release modifier of polymer and $0.75 \% \mathrm{w} / \mathrm{v}$ of PVA used to prepare blank microspheres. The effect of the above process variables is given in table 2.

At low stirring speed, the harder globules of the polymer formed due to coalescence and aggregation while at higher speed the microspheres breakdown to form irregular particles. At $40^{\circ} \mathrm{C}$ temperature, the solvent diffuses into aqueous phase at an intermittent rate and provide sufficient time for droplet formation and hardening of hollow microspheres. The longer the stirring time will provide enough time for the solvent to diffuse and thus improve the yield. For this investigation we found that optimized conditions for preparation of hollow microspheres were stirring rate of 350 $\mathrm{rpm}$, stirring time of $2 \mathrm{~h}$ and system temperature $40^{\circ} \mathrm{C}$.

Table 2: Effect of process variables

\begin{tabular}{lll}
\hline Process conditions & \% Yield & Physical appearance \\
\hline Stirring Rate (rpm) & & \\
150 & 52.25 & Large particles \\
350 & 57.00 & Spherical particles \\
900 & 54.76 & Irregular shape \\
System Temperature & & \\
Room temperature & 50.43 & Soft brittle particle \\
$40^{\circ} \mathrm{C}$ & 57.26 & Spherical particles \\
$60^{\circ} \mathrm{C}$ & 56.04 & Irregularly shaped particles \\
Stirring Time & & \\
30 min. & - & Sticky Lumps \\
$1 \mathrm{~h}$ & 53.79 & Slightly sticky particles \\
$2 \mathrm{~h}$ & 58.04 & Spherical particles \\
\hline
\end{tabular}

\section{Physico-chemical analysis}

The change in frequency and bandwidth of different interacting groups in the spectrum of the mixture of drug and excipients are studied by FTIR analysis [33]. The FT-IR spectral bands of itraconazole, ethylcellulose, Eudragit E100, placebo microspheres and optimized drug loaded formulation are shown in fig. 1. On the interpretation of spectra, it is clear that all characteristic peaks of the drug are intact. Hence, no major shift or addition of new peaks indicated that no significant chemical interaction between drug and polymers.

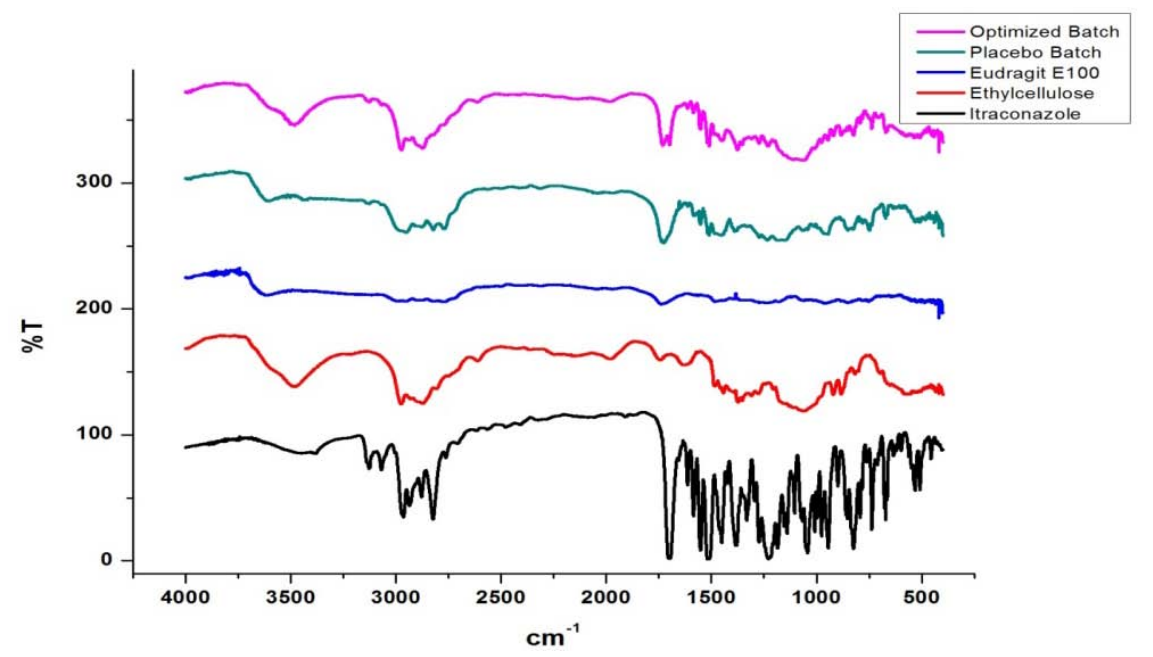

Fig. 1: FTIR spectra of itraconazole; ethylcellulose; eudragit E100; placebo batch and optimized batch

DSC and XRD analysis were carried out to study the change in the physical state of drug and/or any significant drug and excipients interactions [34]. Fig. 2 shows DSC thermograms of itraconazole, ethylcellulose, Eudragit E100, physical mixture and optimized batch. The DSC thermogram of itraconazole shows a sharp endothermic peak at $167.8{ }^{\circ} \mathrm{C}$. Thermograms of ethyl cellulose and Eudragit E100 showed a broad endothermic peak at $56.77{ }^{\circ} \mathrm{C}$ and $65.36{ }^{\circ} \mathrm{C}$ respectively, which represent the melting of polymers. The endothermic peak of itraconazole appears in drug-excipients physical mixture with a slight change in temperature which indicates the crystalline nature of the drug and the absence of any chemical interaction at solid-state. The DSC results of an optimized batch of microspheres showed a small blunt endothermic peak of itraconazole was observed at $161.32{ }^{\circ} \mathrm{C}$, due to a reduction in the crystallinity of drug by its solubilization in the polymer matrix. 


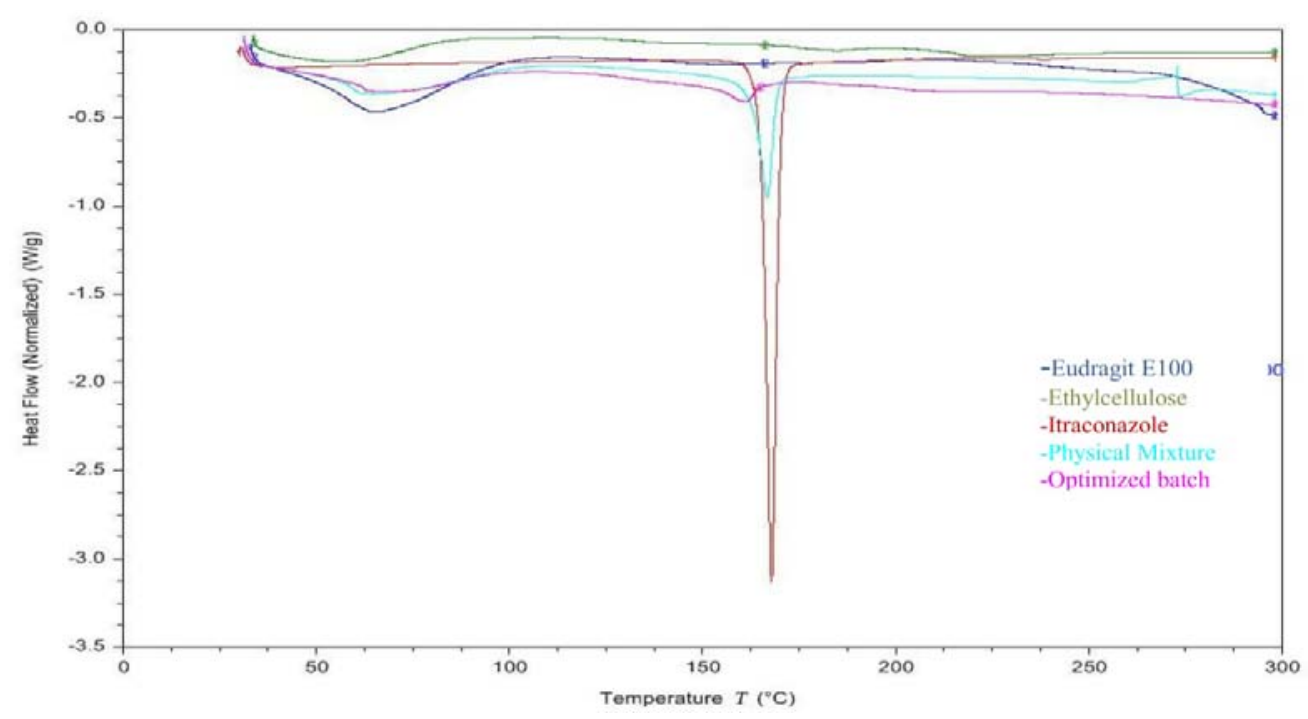

Fig. 2: DSC thermogram of itraconazole; ethylcellulose; eudragit E100; physical mixture and optimized batch

The X-ray diffraction pattern shown in fig. 3, the pure drug showed a range of sharp and distinctive peaks at $2 \theta$ angle of $23.58^{\circ}, 20.46^{\circ}, 17.58^{\circ}$ and $14.58^{\circ}$, having maximum peak intensity at $2 \theta$ angle of $20.46^{\circ}$ indicating crystalline nature of the drug. The physical mixture showed peaks of itraconazole. The DSC results were confirmed by diffractograms of the optimized batch which exemplify the absence of sharp distinctive peaks of itraconazole.

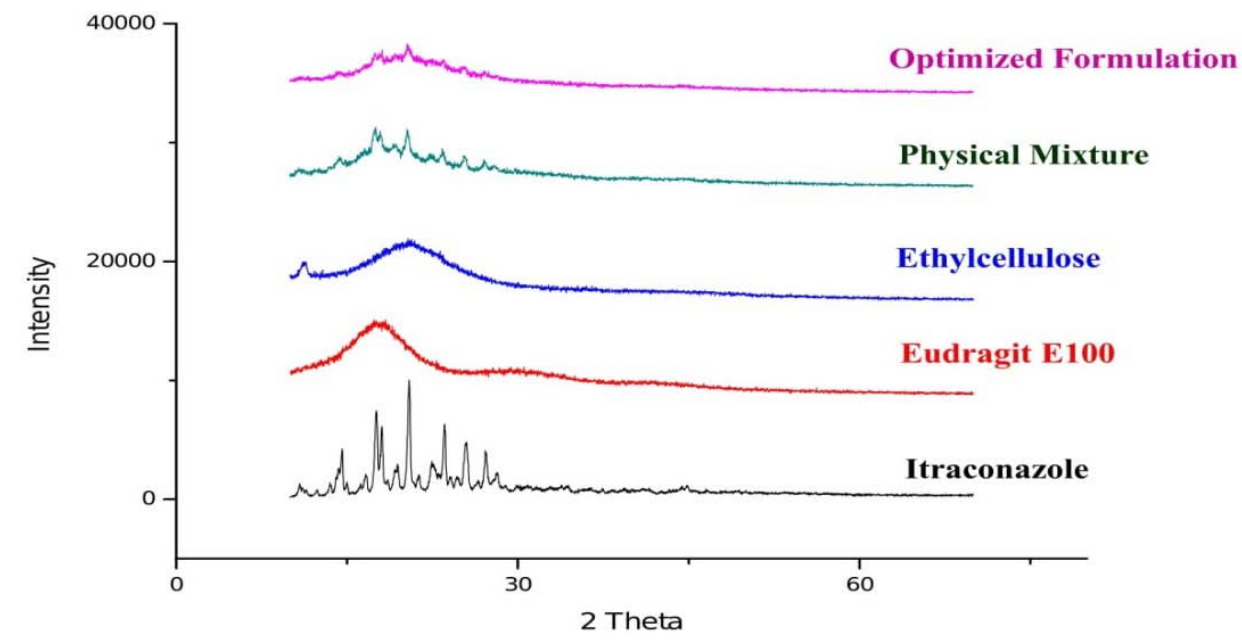

Fig. 3: X-ray diffractograms of itraconazole; ethylcellulose; eudragit E100; physical mixture and optimized batch

\section{Optimization of data analysis}

In the present study, the central composite experimental design was employed as it adequately described the interaction between the factors with the least number of experimental trial runs. The summary of the experimental data and observed responses were given in table 1.

All Observed responses were fitted to various polynomial models. It was seen that the particle size $\left(R_{1}\right)$ and percent cumulative drug release $\left(R_{4}\right)$ fitted best into linear response surface model ( $p$-value 0.0004 for $\mathrm{R}_{1}, p$ value $<0.0001$ for $R_{4}$ ) while the entrapment efficiency $\left(R_{2}\right)$ and percentage buoyancy $\left(\mathrm{R}_{3}\right)$ was found to be fitted best into quadratic response surface model ( $p$-value 0.0004 for $\mathrm{R}_{2}, p$-value 0.0002 for $\mathrm{R}_{3}$ ) with no transformations of data. The polynomial equations generated for responses in terms of coded values given below:

$\mathrm{R}_{1}(\mu \mathrm{m})=389.4+45.4023 \mathrm{X}_{1}+-55.2896 \mathrm{X}_{2}$

$\mathrm{R}_{2}(\%)=74.866+1.49696 \mathrm{X}_{1}+-3.96283 \mathrm{X}_{2}+-0.8525 \mathrm{X}_{1} \mathrm{X}_{2}+4.44887$ $\mathrm{X}_{1}^{2}+5.57387 \mathrm{X}_{2}^{2}$
R3 $(\%)=54.558+7.79616 \quad X_{1}+-7.21136 \quad X_{2}+0.93 \quad X_{1} X_{2}+3.7835$ $\mathrm{X}_{1}^{2}+3.9185 \mathrm{X}_{2}^{2}$

$\mathrm{R}_{4}(\%)=85.2892+-6.07934 \mathrm{X}_{1}+3.16383 \mathrm{X}_{2}$

The polynomial equations demonstrate the relationship between the process variables and response variables. A model was considered to be significant if the P-value $<0.05$. The synergistic effect and antagonistic effect can be illustrated from the positive and negative signs respectively. The sign and magnitude of factor value have a relative impact on the responses. Table 3 showed the result of ANOVA analysis on models indicate that the F-value of response surface model for all responses is significant $(\mathrm{P}<0.05)$ with non-significant 'lack of fit' $(\mathrm{P}>0.05)$ which ensure the reliability of the applied model. The predicted $\mathrm{R}^{2}$ and adjusted $\mathrm{R}^{2}$ were in good agreement which signifies the reliability of models. In addition to the above, adequate precision measure signal to noise ratio, higher values of adequate precision $(>4)$ indicate that developed models are fit to navigate the design space. 
Table 3: Analysis of variance statistics of response surface models

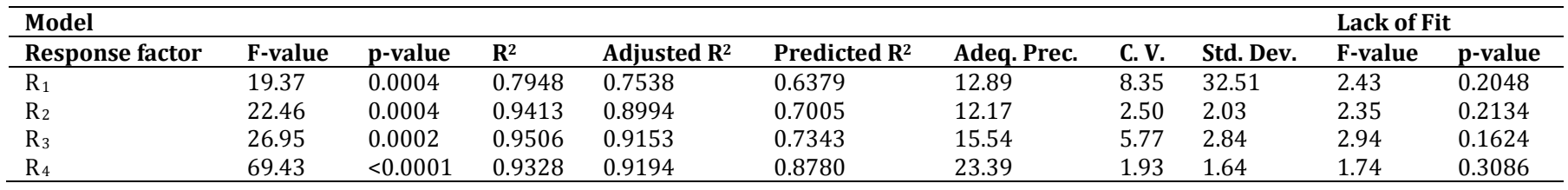

From the polynomial equations, the contour plots and 3D response surface graphs were generated (displayed in fig. 4) signifies that the polymer ratio and concentration of PVA carry a significant effect on particle size, entrapment efficiency, buoyancy, and drug release.

Fig. 4 (a) showed a decrease in particle size in an almost linear manner with an increase in the concentration of PVA as it reduces the interfacial tension between the particles and stabilizes the polymer droplets in the aqueous phase which result in coalescence of particles. The increase in the concentration of ethylcellulose in the ratio of polymers, the particle size increases in linearly. The maximum particle size observed at a high ratio of ethylcellulose and a lower concentration of PVA.

Fig. 4 (b) depicted that the drug entrapment efficiency significantly increased with increase in the ratio of ethylcellulose concentration while Eudragit E100 tends to decrease the entrapment of drug as it increases the permeability of microspheres which leads to diffusion of drug towards external phase [20]. On the other hand, the concentration of PVA has a pronounced effect on entrapment efficiency, as it decreases with increase in the concentration of PVA. However, the influence of the concentration of PVA is more significant than that of the ratio of polymers.

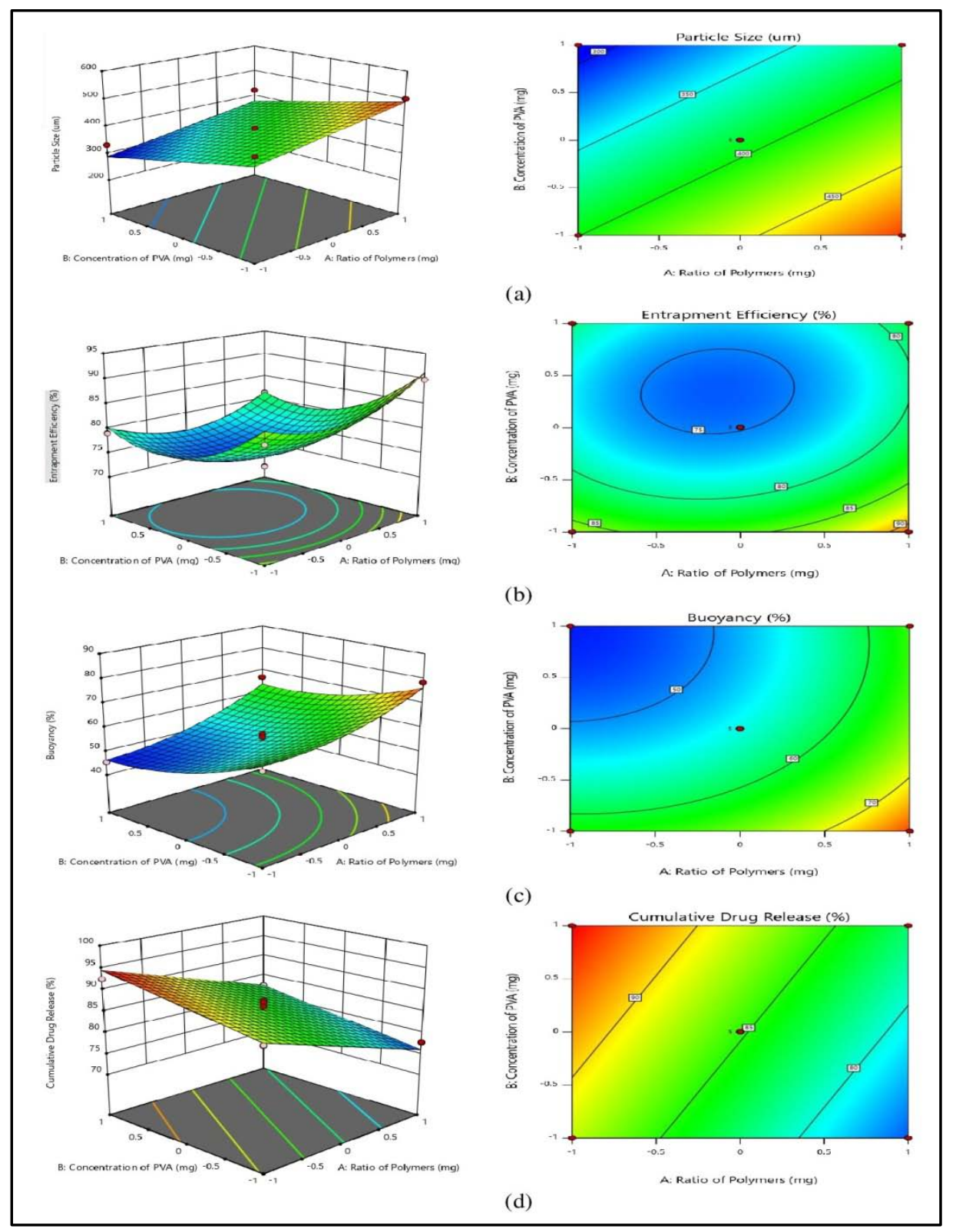

Fig. 4: Two-dimensional contour plots and three-dimensional response surface plots showing the effect of polymers ratio and concentration of PVA on (a) Particle Size; (b) Entrapment efficiency; (c) Buoyancy and (d) Cumulative drug release 

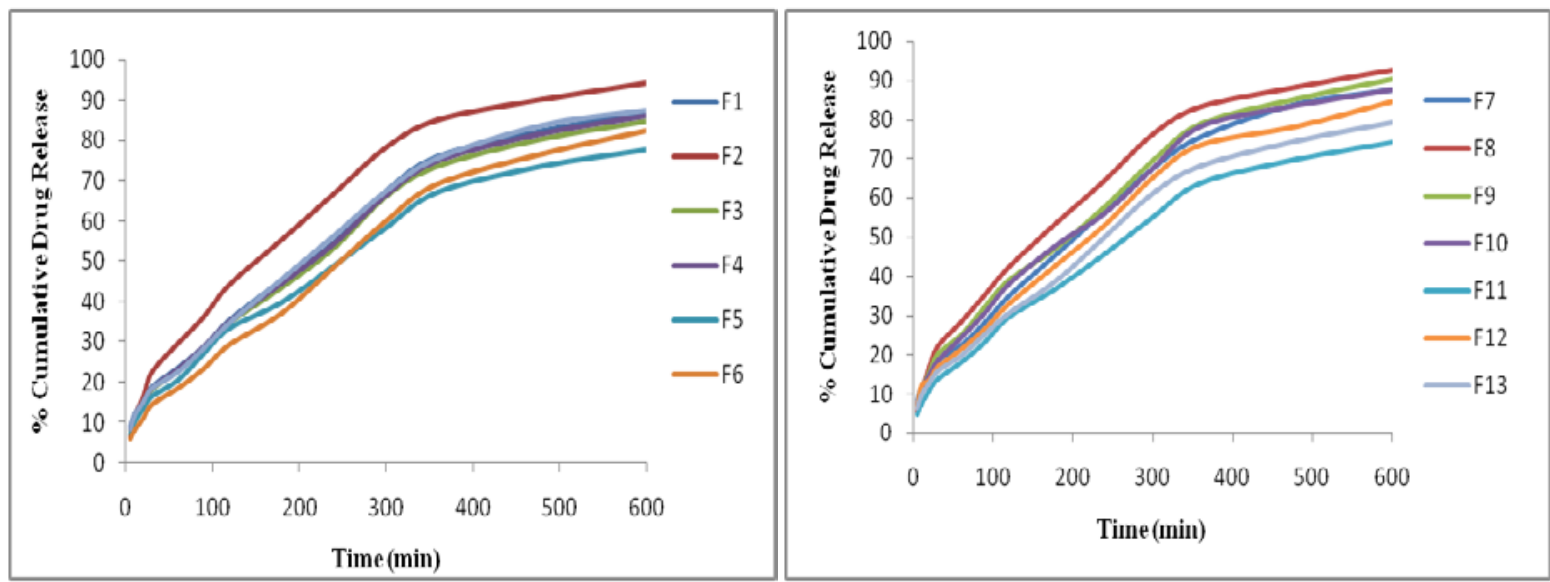

Fig. 5: In vitro dissolution profile of formulations F1-F13

The effect of process variables on buoyancy is shown in fig. 4(c). The buoyancy signifies the floating ability of microspheres owing to their hollow structure. The density of polymers and the size of particles play an important role in buoyancy [35]. As the size of the particle increases the density decreases which directly increase the floating ability of microspheres. Hence, increase in buoyancy can be directly related to increase in polymer ratio especially with the increase in the concentration of ethylcellulose, a low-density hydrophobic polymer but inversely related to increasing in the concentration of PVA as increased concentration of surfactant decrease the particle size which directly decreases the buoyancy. Similarly, the buoyancy of microspheres also decreases up to some extent with the increase in Eudragit E100 polymer due to dissolution and ingression of simulated gastric fluid into microspheres [19].

The response surface plot of percent cumulative drug release shown in fig. 4(d). The finding from in vitro drug release study states the biphasic release pattern of formulations with initial burst release due to surface-associated drug particles followed by slow diffusion of the drug from the polymer matrix, as displayed in fig. 5. It was observed that the drug release increases with the increase in the concentration of Eudragit E100, because of soluble nature of Eudragit E100 polymer in simulated gastric fluid. Concerning the effect of emulsifier, PVA resulted in a significant increase in the release rate. This can be explained by the relative increase in surface area by the reduction in particle size and it also increases the wet ability of particles which subsequently results in increased drug release [36].

Floating microspheres were subjected to numerical optimization tool along with desirability approach of Design-Expert software (Version 11.0.0, Stat-Ease Inc., Minneapolis, MN). The goal is to obtain the optimum values of the independent variables. Optimization was performed and the optimal calculated parameters adjudged were having the ratio of polymers $\left(\mathrm{X}_{1}\right) \quad 2: 1$ and concentration of PVA $\left(\mathrm{X}_{2}\right) 0.5 \% \mathrm{w} / \mathrm{v}$ with the highest desirability. The results experimental and predicted responses for final optimized concentration are tabulated in table 4.

Table 4: Comparison of experimental results with predicted values of optimized formulation

\begin{tabular}{lllll}
\hline $\mathbf{X}_{\mathbf{1}} / \mathbf{X}_{\mathbf{2}}$ & Response & Experimental & Predicted & \%Error \\
\hline & $\mathrm{R}_{1}: 1 / 0.5$ & 432.1 & 399.28 & 8.21 \\
& $\mathrm{R}_{2}$ & 84.11 & 86.50 & -2.76 \\
& $\mathrm{R}_{3}$ & 60.77 & 62.60 & -2.92 \\
& $\mathrm{R}_{4}$ & 87.72 & 88.20 & -0.54 \\
\hline
\end{tabular}

\section{SEM analysis}

SEM observed the shape and morphology of microspheres as shown in fig. 6. The analysis confirmed the smooth and spherical shape of particles. The microsphere shell also showed some small pore over it and having an internal hollow cavity. The pore over the surface may be due to the diffusion of solvent and the central hollow cavity formed by the generation of gaseous phase inside the polymer droplet by the evaporation of entrapped solvent inside the microspheres.

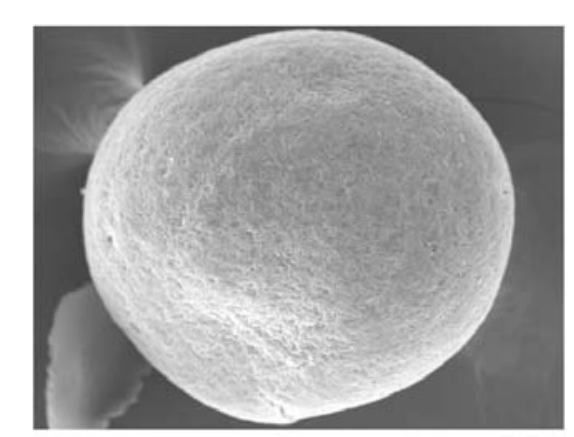

(a)

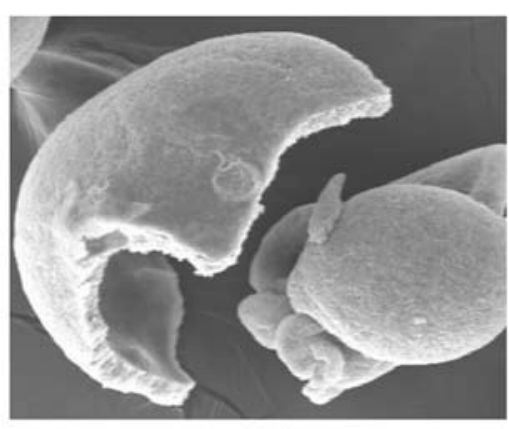

(b)

Fig. 6: SEM image of the surface view of a hollow microsphere; (b) SEM image showing central hollow cavity 


\section{Drug release kinetics}

In order to determine the mechanism of drug release profile of optimized batch, the in vitro release data was subjected to various mathematical kinetic models and regression coefficient value after interpretation of data comes out to be 0.936 for zero-order, 0.990 for first order, 0.989 for Higuchi's model and 0.994 for Korsmeyer and Peppa's model and drug release exponent (n) comes out to be 0.531 . The mechanism of drug release was found to be diffusion and erosion controlled as the regression coefficient value is maximum for Korsmeyer and Peppa's model [29].

\section{Stability studies}

All the parameters studied for the stability of optimized formulation were found to be satisfying at the end of six months (table 5). The formulation showed no significant change in properties over time at different temperature and humidity conditions. The microspheres remained well within the acceptance criteria during the proposed study at accelerated conditions. Hence, floating microspheres are chemically and physically stable and retain their pharmaceutical properties for over 6 mo.

Table 5: Stability study profile

\begin{tabular}{|c|c|c|c|c|c|}
\hline Parameters & 0 mo & $1 \mathrm{mo}$ & $2 \mathrm{mo}$ & $3 \mathrm{mo}$ & $6 \mathrm{mo}$ \\
\hline \multicolumn{6}{|c|}{ Refrigerator conditions $\left(5 \pm 3^{\circ} \mathrm{C}\right)$} \\
\hline Visual Appearance & White discrete particles & No physical change & No physical change & No physical change & No physical change \\
\hline Drug Content & $17.72 \pm 0.30$ & $17.70 \pm 0.12$ & $17.68 \pm 0.15$ & $17.67 \pm 0.20$ & $17.33 \pm 0.25$ \\
\hline Buoyancy & $60.77 \pm 1.02$ & $60.73 \pm 0.75$ & $60.65 \pm 0.80$ & $60.5 \pm 1.05$ & $60.00 \pm 1.25$ \\
\hline In vitro drug release & $87.72 \pm 1.23$ & $87.64 \pm 0.78$ & $87.57 \pm 0.90$ & $87.4 \pm 1.24$ & $87.25 \pm 1.40$ \\
\hline \multicolumn{6}{|c|}{ Room temperature $\left(25 \pm 2^{\circ} \mathrm{C}\right)$} \\
\hline Visual Appearance & White discrete particles & No physical change & No physical change & No physical change & No physical change \\
\hline Drug Content & $17.72 \pm 0.30$ & $17.61 \pm 0.23$ & $17.48 \pm 0.49$ & $17.24 \pm 0.41$ & $16.70 \pm 0.52$ \\
\hline Buoyancy & $60.77 \pm 1.02$ & $60.65 \pm 0.95$ & $60.40 \pm 1.12$ & $60.15 \pm 1.24$ & $59.80 \pm 1.06$ \\
\hline In vitro drug release & $87.72 \pm 1.23$ & $87.50 \pm 0.87$ & $87.25 \pm 0.76$ & $86.75 \pm 1.12$ & $86.22 \pm 1.52$ \\
\hline \multicolumn{6}{|c|}{ Accelerated conditions $\left(40 \pm 2{ }^{\circ} \mathrm{C} / 75 \pm 5 \% \mathrm{RH}\right)$} \\
\hline Visual Appearance & White discrete particles & No physical change & No physical change & No physical change & Small aggregates formed \\
\hline Drug content & $17.72 \pm 0.30$ & $17.35 \pm 0.28$ & $17.12 \pm 0.54$ & $16.85 \pm 0.38$ & $16.00 \pm 0.44$ \\
\hline Buoyancy & $60.77 \pm 1.02$ & $60.50 \pm 0.50$ & $60.15 \pm 1.08$ & $59.95 \pm 0.95$ & $59.50 \pm 1.24$ \\
\hline In vitro drug release & $87.72 \pm 1.23$ & $87.53 \pm 1.12$ & $87.15 \pm 0.70$ & $86.54 \pm 0.85$ & $85.45 \pm 1.44$ \\
\hline
\end{tabular}

All values are the mean $\pm \operatorname{SD}(n=3)$

\section{CONCLUSION}

Itraconazole-loaded non-effervescent floating hollow microspheres were successfully prepared by emulsion-solvent diffusion evaporation technique employing optimal process conditions. The central composite design of response surface methodology approach is a very useful statistical technique to conclude the effect of independent variables on the responses. The prepared microspheres characterize by particle size, percent entrapment efficiency, in vitro floating ability and in vitro drug release, and were observed that the formulations were significantly affected by the ratio of lowdensity polymer and release modifier and concentration of PVA. The result of the stability study depicted that the floating microspheres were found to be stable for 6 mo. The presence of PVA as an emulsifier and pH-dependent Eudragit E 100 as a release modifying polymer facilitate significant drug release in simulated gastric fluid, the desired medium for drug absorption. Besides, the drug release kinetics follows Korsmeyer Peppas model as the drug release occurs by diffusion and erosion controlled mechanism. The hollow microspheres with a release modifying polymer is a useful approach to retain the formulation at its absorption site and facilitate the drug release.

\section{ACKNOWLEDGMENT}

The authors express gratitude to Department of Science and Technology, New Delhi, for providing financial assistance to Surbhi Rohilla (IF150924) under "INSPIRE" fellowship and to SAIF, AIIMS, New Delhi for SEM analysis. Department of Pharmaceutical Sciences, UHS, Rohtak for particle size analysis.

\section{AUTHORS CONTRIBUTIONS}

All the authors have contributed equally

\section{CONFLICT OF INTERESTS}

Declared none

\section{REFERENCES}

1. Hong JY, Kim JK, Song YK, Park JS, Kim CK. A new selfemulsifying formulation of itraconazole with improved dissolution and oral absorption. J Controlled Release 2006;110:332-8.

2. Prentice AG, Glasmacher A. Making sense of itraconazole pharmacokinetics. J Antimicrob Chemother 2005;56(Suppl 1):i17-22.

3. Domínguez Gil Hurle A, Sanchez Navarro A, Garcia Sanchez MJ. Therapeutic drug monitoring of itraconazole and the relevance of pharmacokinetic interactions. Clin Microbiol Infect 2006;12:97-106.

4. Darwazeh AM, Darwazeh TA. What makes oral candidiasis recurrent infection? a clinical view. Mycologia 2014 http://dx.doi.org/10.1155/2014/758394

5. Kim JY, Rhee YS, Park CW, Ha JM, Park ES. Preparation and evaluation of dual-mode floating gastroretentive tablets containing itraconazole. Drug Delivery 2014;21:519-29.

6. Madgulkar A, Kadam S, Pokharkar V. Studies on formulation development of mucoadhesive sustained-release itraconazole tablet using response surface methodology. AAPS PharmSciTech 2008;9:998.

7. Thakkar HP, Khunt A, Dhande RD, Patel AA. Formulation and evaluation of Itraconazole nanoemulsion for enhanced oral bioavailability. J Microencapsulation 2015;32:559-69.

8. Zhong Y, Jing G, Tian B, Huang $\mathrm{H}$, Zhang Y, Gou J, et al. Supersaturation induced by Itraconazole/Soluplus ${ }^{\circledR}$ micelles provided high GI absorption in vivo. Asian J Pharm 2016;11:255-64.

9. Yi Y, Yoon HJ, Kim BO, Shim M, Kim SO, Hwang SJ, et al. A mixed polymeric micellar formulation of itraconazole: characteristics, toxicity, and pharmacokinetics. J Controlled Release 2007;117:59-67.

10. Türkmen B, Gökbulut E, Ozdemi N. A new approach to enhance bioavailability: dosage forms with extended residence time in the stomach. FABAD J Pharm Sci 2017;42:9.

11. Rao MR, Borate SG, Thanki KC, Ranpise AA, Parikh GN. Development and in vitro evaluation of floating rosiglitazone maleate microspheres. Drug Dev Ind Pharm 2009;35:834-42.

12. Kohli S, Sharma M, Pal A. Ethylcellulose floating microspheres of antidiabetic agent: in vitro and in vivo evaluation. Int J Appl Pharm 2016;9:44-9. 
13. Panwar MM. Factorial design approach for optimization of floating microspheres of diltiazem hydrochloride. Asian J Pharm 2015;9:206-12.

14. Murtaza G. Ethylcellulose microparticles: a review. Acta Pol Pharm 2012;69:11-22.

15. Mastiholimath VS, Dandagi PM, Gadad AP, Mathews R, Kulkarni AR. In vitro and in vivo evaluation of ranitidine hydrochloride ethyl cellulose floating microparticles. J Microencapsulation 2008;25:307-14.

16. Vaghani S, Vasanti S, Chaturvedi K, Satish CS, Jivani NP. Stomach-specific drug delivery of 5-fluorouracil using ethylcellulose floating microspheres. Pharm Dev Technol 2010;15:154-61.

17. Assas N, Elbahri Z, Baitiche M, Djerboua F. Effects of some process parameters on the niflumic acid controlled release polymeric microspheres: optimization using designs of experiments. Asia Pacific J Chem Eng 2019:e2283. https://doi.org/10.1002/apj.2283

18. Emeje MO, Kunle 00, Ofoefule SI. Compaction characteristics of ethylcellulose in the presence of some channeling agents. AAPS PharmSciTech 2006;7:E18-21.

19. Farooq U, Khan S, Nawaz S, Ranjha NM, Haider MS, Khan MM, et al. Enhanced gastric retention and drug release via development of novel floating microspheres based on Eudragit E100 and polycaprolactone: synthesis and in vitro evaluation. Des Monomers Polym 2017;20:419-33.

20. Kawashima Y, Niwa T, Takeuchi H, Hino T, Itoh Y. Hollow microspheres for use as a floating controlled drug delivery system in the stomach. J Pharm Sci 1992;81:135-40.

21. El-Kamel AH, Sokar MS, Al Gamal SS, Naggar VF. Preparation and evaluation of ketoprofen floating oral delivery system 1 . Int J Pharm 2001;220:13-21.

22. Junyaprasert VB, Pornsuwannapha S. Floating properties and release characteristics of hollow microspheres of acyclovir. Drug Delivery 2008;15:331-41.

23. Kawashima Y, Niwa T, Takeuchi H, Hino T, Ito Y. Preparation of multiple unit hollow microspheres (microballoons) with acrylic resin containing tranilast and their drug release characteristics (in vitro) and floating behavior (in vivo). J Controlled Release 1991;16:279-89.

24. Sato Y, Kawashima Y, Takeuchi H, Yamamoto H. Physicochemical properties to determine the buoyancy of hollow microspheres (microballoons) prepared by the emulsion solvent diffusion method. Eur J Pharm Biopharm 2003;55:297-304

25. Gaur PK, Mishra S, Bajpai M. Formulation and evaluation of controlled-release of telmisartan microspheres: in vitro/in vivo study. J Food Drug Anal 2014;22:542-8.

26. El Nashar NF, Donia AA, Mady OY, El Maghraby GM. Formulation of clarithromycin floating microspheres for eradication of Helicobacter pylori. J Drug Delivery Sci Technol 2017;41:213-21.

27. Najmuddin M, Ahmed A, Shelar S, Patel V, Khan T. Floating microspheres of ketoprofen: formulation and evaluation. Int J Pharm Pharm Sci 2010;2:83-7.

28. Dash S, Murthy PN, Nath L, Chowdhury P. Kinetic modeling on drug release from controlled drug delivery systems. Acta Pol Pharm 2010;67:217-3

29. Costa P, Lobo JM. Modeling and comparison of dissolution profiles. Eur J Pharm Sci 2001;13:123-33.

30. Matthews BR. Regulatory aspects of stability testing in Europe. Drug Dev Ind Pharm 1999;25:831-56.

31. Pandit V, Pai RS, Yadav V, Devi K, Surekha BB, Inamdar MN Suresh S. Pharmacokinetic and pharmacodynamic evaluation of floating microspheres of metformin hydrochloride. Drug Dev Ind Pharm 2013;39:117-27.

32. Sharma M, Kohli S, Pal A. Stability of floating microspheres at normal and accelerated conditions. Int J Pharm Pharm Sci 2016;8:452-4

33. Mohamed AI, Abd-Motagaly AM, Ahmed OA, Amin S, Mohamed Ali AI. Investigation of drug-polymer compatibility using chemometric-assisted UV-spectrophotometry. Pharmaceutics 2017;9:7.

34. Dinte EL, Bodoki E, Leucuta S, Iuga CA. Compatibility studies between drugs and excipients in the preformulation phase of buccal mucoadhesive systems. Farmacia 2013;61:703-12.

35. Sato $Y$, Kawashima $Y$, Takeuchi H, Yamamoto H. In vitro evaluation of floating and drug-releasing behaviors of hollow microspheres (microballoons) prepared by the emulsion solvent diffusion method. Eur J Pharm Biopharm 2004;57:23543.

36. Pachuau L, Mazumder B. A study on the effects of different surfactants on Ethylcellulose microspheres. Int J Pharm Tech Res 2009;1:966-71. 